\title{
Synthesis and Radical Copolymerization of Novel Phenyl-Disubstituted Propyl Cyanoacrylates
}

\author{
Christopher Savittieri R, Shechinah Tinsley M, Andrew Diehn J, Fatema Hai, Kara Humanski E, Eric Kempke J, \\ Benjamin Killam Y, Jimmy Kozeny, Evana Makhoul W, Molly Obert C, Anthony Parisi C, Victoria Parrilli C and \\ Gregory Kharas B*
}

Department of Chemistry, DePaul University, USA

Submission: May 06, 2019; Published: May 13, 2019

*Corresponding author: Gregory Kharas B, Department of Chemistry, DePaul University, Chicago, IL, USA

Abstract

Novel phenyl-disubstituted propyl 3-(R-phenyl)-2-cyanoacrylates, $\mathrm{RPhCH}=\mathrm{C}(\mathrm{CN}) \mathrm{CO}_{2} \mathrm{C}_{3} \mathrm{H}_{7}$ (where $\mathrm{R}$ is 2-fluoro-5-methyl, 3-iodo-4-methoxy, 5-iodo-2-methoxy, 3,5-dichloro, 3,4-difluoro, 3,5-difluoro, 2-chloro-4-fluoro, 2-chloro-6-fluoro, 3-chloro-2-fluoro, and 3-chloro-4-fluoro) were prepared using condensation of substituted benzoic aldehydes and propyl ester of cyanoacetic acid. The ethynyl benzene copolymerization of novel cyanoacrylates was conducted in solution with radical initiation at $70{ }^{\circ} \mathrm{C}$. Nitrogen analysis, ${ }^{1} \mathrm{H} \&{ }^{13} \mathrm{C}$ NMR was used to analyze composition and the structure. Thermal behavior of the copolymers was analyzed by DSC and TGA.

Keywords: Radical copolymerization; Styrene copolymers; Trisubstituted ethylene’s; Cyanoacrylates

\section{Introduction}

Ring-functionalized trisubstituted ethylene's (TSE), esters of 3-phenyl-2-cyanoacrylates, $\mathrm{R}^{1} \mathrm{PhCH}=\mathrm{C}(\mathrm{CN}) \mathrm{CO}_{2} \mathrm{R}^{2}$ continue to attract attention as compounds with interesting properties and as comonomers for modification of commercial polymers. 3,4-Difluorophenyl substituted methyl 3-phenyl-2-cyanoacrylate was used in synthesis and studies of histamine $\mathrm{H}_{2}$ agonistic activity [1]. Ethyl 3-phenyl-2-cyanoacrylate was used in studies of stereoselective cascade assembling of $\left(1 \mathrm{R}^{*}, 2 \mathrm{~S}^{*}\right)-1$-cyano-5,7dialkyl-4,6,8-trioxo-2-aryl-5,7-diazaspiro[2.5]octane-1-carboxylates [2] and analgesic activity of 6-fluoroindan-1-carboxylic acid [3]. Alkyl 2-cyanoacrylates are a family of vinyl monomers renowned for their high reactivity, instant adhesive properties, and wide-ranging applications [4].

We have reported synthesis and ethynylbenzene copolymerization of similar ring-substituted methyl [5], ethyl [6], \& butyl 3-phenyl-2-cyanoacrylates [7]. With the objective to design novel structures, that could serve as a spring board for further development of novel materials with new properties and applications, we have prepared halogen ring-disubstituted propyl 3-phenyl-2-cyanoacrylate (PPCA), $\mathrm{RPhCH}=\mathrm{C}(\mathrm{CN}) \mathrm{CO}_{2} \mathrm{C}_{3} \mathrm{H}_{7}$, where $\mathrm{R}$ is 2-fluoro-5-methyl, 3-iodo-4-methoxy, 5-iodo-2-methoxy, 3,5-dichloro, 3,4-difluoro, 3,5-difluoro, 2-chloro-4-fluoro, 2-chloro-6-fluoro, 3-chloro-2-fluoro, and 3-chloro-4-fluoro, and copolymerize them with ethynylbenzene. To the best of our knowledge, there have been no reports on either synthesis of these propyl phenyl cyanoacrylates, nor their copolymerization with ethynylbenzene.

\section{Experimental}

All benzoic aldehydes, propyl cyanoacetate, piperidine, ethynylbenzene, 1,1'-azobiscyclohexanecarbonitrile, (ABCN), and toluene supplied from Sigma-Aldrich Co., were used as received. The melting points and $\mathrm{T}_{\mathrm{g}}$, were measured with TA Model Q10 DSC. The thermal stability of the copolymers was measured by TGA TA Model Q50 from ambient temperature to $800{ }^{\circ} \mathrm{C}$ at $20{ }^{\circ} \mathrm{C} / \mathrm{min}$. The MW of the copolymers was determined by GPC using an Altech 426 HPLC pump at an elution rate of $1.0 \mathrm{~mL} / \mathrm{min}$; Phenogel $5 \mu$ Linear column at $25 \stackrel{\circ}{\circ} \mathrm{C}$ and Viscotek 302 detector. ${ }^{1} \mathrm{H} \&{ }^{13} \mathrm{C}$ NMR spectra were obtained on $10-25 \%$ $(\mathrm{w} / \mathrm{v})$ monomer or polymer solutions in $\mathrm{CDCl}_{3}$ at ambient temperature using Avance $300 \mathrm{MHz}$ spectrometer. Elemental analyses were performed by Midwest Microlab, LLC (IN).

\section{Results and Discussion}

\section{PPCA synthesis}

The ring-substituted propyl 3-phenyl-2-cyanoacrylates (PPCA) were synthesized by Knoevenagel condensation [8] of 
a ring-substituted benzoic aldehyde with propyl cyanoacetate, catalyzed by base, piperidine (1).

$\mathrm{RphCHO}+\mathrm{NCCH}_{2} \mathrm{CO}_{2} \mathrm{C}_{3} \mathrm{H}_{7} \rightarrow \mathrm{RphCH}=\mathrm{C}(\mathrm{CN}) \mathrm{CO}_{2} \mathrm{C}_{3} \mathrm{H}_{7}$

Where $\mathrm{R}$ is 2-fluoro-5-methyl, 3-iodo-4-methoxy, 5-iodo2-methoxy, 3,5-dichloro, 3,4-difluoro, 3,5-difluoro, 2-chloro4-fluoro, 2-chloro-6-fluoro, 3-chloro-2-fluoro, \& 3-chloro-4fluoro. The preparation procedure was essentially the same for all the monomers. In a typical synthesis, equimolar amounts of propyl cyanoacetate and an appropriate ring-substituted benzoic aldehyde were mixed in equimolar ratio in a $20 \mathrm{~mL}$ vial. A few drops of piperidine were added with stirring. The product of the reaction was isolated by filtration and purified by crystallization from 2-propanol. The condensation reaction proceeded smoothly, yielding products, which were purified by conventional techniques.

\section{Propyl 3-(2-fluoro-5-methylphenyl)-2-cyanoacrylate}

Yield 87\%; ${ }^{1} \mathrm{H}-\mathrm{NMR} \delta 8.4(\mathrm{~s}, 1 \mathrm{H}, \mathrm{CH}=), 8.2-6.9(\mathrm{~m}, 3 \mathrm{H}, \mathrm{Ph})$, $4.3\left(\mathrm{t}, 2 \mathrm{H}, \mathrm{OCH}_{2}\right), 2.3(\mathrm{~s}, 3 \mathrm{H}, \mathrm{CH} 3), 1.7\left(\mathrm{~m}, 2 \mathrm{H}, \mathrm{OCH}_{2} \mathrm{CH}_{2}\right), 1.0(\mathrm{t}$, $\left.3 \mathrm{H}, \mathrm{OCH}_{2} \mathrm{CH}_{2} \mathrm{CH}_{3}\right) ;{ }^{13} \mathrm{C}-\mathrm{NMR} \delta 163(\mathrm{C}=0), 152(\mathrm{HC}=), 138,135$, 130, 121, $115(\mathrm{Ph}), 116(\mathrm{CN}), 104(\mathrm{C}=), 67\left(\mathrm{OCH}_{2}\right), 22\left(\mathrm{OCH}_{2} \mathrm{CH}_{2}\right)$, $21\left(\mathrm{CH}_{3}\right), 10\left(\mathrm{OCH}_{2} \mathrm{CH}_{2} \mathrm{CH}_{3}\right)$; Anal. Calcd. for $\mathrm{C}_{14} \mathrm{H}_{14} \mathrm{FNO}_{2}$ : C, 68.00; H, 5.71; N, 5.66; Found: C, 67.85; H, 5.75; N, 5.86.

\section{Propyl 3-(3-iodo-4-methoxyphenyl)-2-cyanoacrylate}

Yield 92\%; mp $140{ }^{\circ} \mathrm{C},{ }^{1} \mathrm{H}-\mathrm{NMR} \quad \delta 8.3(\mathrm{~s}, 1 \mathrm{H}, \mathrm{CH}=), 8.2-6.7$ $(\mathrm{m}, 3 \mathrm{H}, \mathrm{Ph}), 4.3\left(\mathrm{t}, 2 \mathrm{H}, \mathrm{OCH}_{2}\right), 4.0\left(\mathrm{~s}, 3 \mathrm{H}, \mathrm{OCH}_{3}\right), 1.8(\mathrm{~m}, 2 \mathrm{H}$, $\left.\mathrm{OCH}_{2} \mathrm{CH}_{2}\right), 1.0\left(\mathrm{t}, 3 \mathrm{H}, \mathrm{OCH}_{2} \mathrm{CH}_{2} \mathrm{CH}_{3}\right) ;{ }^{13} \mathrm{C}-\mathrm{NMR} \delta 163(\mathrm{C}=0)$, $154(\mathrm{HC}=), 128,118,88(\mathrm{Ph}), 116(\mathrm{CN}), 100(\mathrm{C}=), 67\left(\mathrm{OCH}_{2}\right)$, $56\left(\mathrm{OCH}_{3}\right), 22\left(\mathrm{OCH}_{2} \mathrm{CH}_{2}\right), 10\left(\mathrm{OCH}_{2} \mathrm{CH}_{2} \mathrm{CH}_{3}\right)$; Anal. Calcd. for $\mathrm{C}_{14} \mathrm{H}_{14} \mathrm{INO}_{3}$ : C, 45.30; H, 3.80; N, 3.77; Found: C, 44.47; H, 3.72; $\mathrm{N}, 3.33$.

\section{Propyl 3-(5-iodo-2-methoxyphenyl)-2-cyanoacrylate}

Yield 95\%; mp $93{ }^{\circ} \mathrm{C},{ }^{1} \mathrm{H}-\mathrm{NMR} \delta 8.2(\mathrm{~s}, 1 \mathrm{H}, \mathrm{CH}=)$, 7.4-6.7 (m, $3 \mathrm{H}, \mathrm{Ph}), 4.3\left(\mathrm{t}, 2 \mathrm{H}, \mathrm{OCH}_{2}\right), 3.9\left(\mathrm{~s}, 3 \mathrm{H}, \mathrm{OCH}_{3}\right), 1.8\left(\mathrm{~m}, 2 \mathrm{H}, \mathrm{OCH}_{2} \mathrm{CH}_{2}\right)$, $1.1\left(\mathrm{t}, 3 \mathrm{H}, \mathrm{OCH}_{2} \mathrm{CH}_{2} \mathrm{CH}_{3}\right) ;{ }^{13} \mathrm{C}-\mathrm{NMR} \delta 163(\mathrm{C}=0), 152(\mathrm{HC}=)$, 122, 115, $80(\mathrm{Ph}), 116(\mathrm{CN}), 111(\mathrm{C}=), 67\left(\mathrm{OCH}_{2}\right), 56\left(\mathrm{OCH}_{3}\right)$, $22\left(\mathrm{OCH}_{2} \mathrm{CH}_{2}\right), 10\left(\mathrm{OCH}_{2} \mathrm{CH}_{2} \mathrm{CH}_{3}\right)$; Anal. Calcd. for $\mathrm{C}_{14} \mathrm{H}_{14} \mathrm{INO}_{3}: \mathrm{C}$, 45.30; H, 3.80; N, 3.77; Found: C, 44.78; H, 3.87; N, 4.05.

\section{Propyl 3-(3,5-dichlorophenyl)-2-cyanoacrylate}

Yield 75\%; mp $123{ }^{\circ} \mathrm{C},{ }^{1} \mathrm{H}-\mathrm{NMR} \delta 8.1$ (s, $1 \mathrm{H}, \mathrm{CH}=$ ), 7.9-7.5 $(\mathrm{m}, 3 \mathrm{H}, \mathrm{Ph}), 4.3\left(\mathrm{t}, 2 \mathrm{H}, \mathrm{OCH}_{2}\right), 1.8\left(\mathrm{~m}, 2 \mathrm{H}, \mathrm{OCH}_{2} \mathrm{CH}_{2}\right), 1.0(\mathrm{t}, 3 \mathrm{H}$, $\left.\mathrm{OCH}_{2} \mathrm{CH}_{2} \mathrm{CH}_{3}\right) ;{ }^{13} \mathrm{C}-\mathrm{NMR} \delta 163(\mathrm{C}=0), 154$ ( $\left.\mathrm{HC}=\right), 135,134$, $128(\mathrm{Ph}), 116(\mathrm{CN}), 103(\mathrm{C}=), 66\left(\mathrm{OCH}_{2} \mathrm{CH}_{2}\right), 22\left(\mathrm{OCH}_{2} \mathrm{CH}_{2}\right), 10$ $\left(\mathrm{OCH}_{2} \mathrm{CH}_{2} \mathrm{CH}_{3}\right)$; Anal. Calcd. for $\mathrm{C}_{13} \mathrm{H}_{11} \mathrm{C}_{12} \mathrm{NO2}$ : C, 54.95; H, 3.90; N, 4.93; Found: C, 54.84; H, 3.97; N, 4.89.

\section{Propyl 3-(3,4-difluorophenyl)-2-cyanoacrylate}

Yield 46\%; mp $74{ }^{\circ} \mathrm{C},{ }^{1} \mathrm{H}-\mathrm{NMR} \delta 8.1(\mathrm{~s}, 1 \mathrm{H}, \mathrm{CH}=)$, 7.9-7.0 $(\mathrm{m}, 3 \mathrm{H}, \mathrm{Ph}), 4.3\left(\mathrm{t}, 2 \mathrm{H}, \mathrm{OCH}_{2}\right), 1.7\left(\mathrm{~m}, 2 \mathrm{H}, \mathrm{OCH}_{2} \mathrm{CH}_{2}\right), 1.0(\mathrm{t}, 3 \mathrm{H}$,
$\left.\mathrm{OCH}_{2} \mathrm{CH}_{2} \mathrm{CH}_{3}\right) ;{ }^{13} \mathrm{C}-\mathrm{NMR} \delta 163(\mathrm{C}=0), 153(\mathrm{HC}=), 150,130$, 125, $118(\mathrm{Ph}), 116(\mathrm{CN}), 109(\mathrm{C}=), 67\left(\mathrm{OCH}_{2}\right), 22\left(\mathrm{OCH}_{2} \mathrm{CH}_{2}\right), 10$ $\left(\mathrm{OCH}_{2} \mathrm{CH}_{2} \mathrm{CH}_{3}\right)$; Anal. Calcd. for $\mathrm{C}_{13} \mathrm{H}_{11} \mathrm{~F}_{2} \mathrm{NO}_{2}: \mathrm{C}, 62.15 ; \mathrm{H}, 4.41 ; \mathrm{N}$, 5.58; Found: C, 61.97; H, 4.62; N, 5.54 .

\section{Propyl 3-(3,5-difluorophenyl)-2-cyanoacrylate}

Yield 82\%; mp $76{ }^{\circ} \mathrm{C},{ }^{1} \mathrm{H}-\mathrm{NMR} \delta 8.1$ (s, $\left.1 \mathrm{H}, \mathrm{CH}=\right)$ ) 7.7-6.7 $(\mathrm{m}, 3 \mathrm{H}, \mathrm{Ph}), 4.3\left(\mathrm{t}, 2 \mathrm{H}, \mathrm{OCH}_{2}\right), 1.8\left(\mathrm{~m}, 2 \mathrm{H}, \mathrm{OCH}_{2} \mathrm{CH}_{2}\right), 1.0(\mathrm{t}, 3 \mathrm{H}$, $\mathrm{OCH}_{2} \mathrm{CH}_{2} \mathrm{CH}_{3}$ ); ${ }^{13} \mathrm{C}-\mathrm{NMR} \delta 162(\mathrm{C}=0), 153$ (HC=), 163, 145, $135(\mathrm{Ph}), 115(\mathrm{CN}), 103(\mathrm{C}=), 67\left(\mathrm{OCH}_{2}\right), 22\left(\mathrm{OCH}_{2} \mathrm{CH}_{2}\right), 10$ $\left(\mathrm{OCH}_{2} \mathrm{CH}_{2} \mathrm{CH}_{3}\right)$; Anal. Calcd. for $\mathrm{C}_{13} \mathrm{H}_{11} \mathrm{~F}_{2} \mathrm{NO}_{2}$ : C, 62.15; H, 4.41; N, 5.58; Found: C, 61.25; H, 4.40; N, 5.79.

\section{Propyl 3-(2-chloro-4-fluorophenyl)-2-cyanoacrylate}

Yield 88\%; mp $80^{\circ} \mathrm{C},{ }^{1} \mathrm{H}-\mathrm{NMR} \delta 8.1$ (s, $\left.1 \mathrm{H}, \mathrm{CH}=\right)$, 7.9-6.7 $(\mathrm{m}, 3 \mathrm{H}, \mathrm{Ph}), 4.3\left(\mathrm{t}, 2 \mathrm{H}, \mathrm{OCH}_{2}\right), 1.7\left(\mathrm{~m}, 2 \mathrm{H}, \mathrm{OCH}_{2} \mathrm{CH}_{2}\right), 1.0(\mathrm{t}, 3 \mathrm{H}$, $\left.\mathrm{OCH}_{2} \mathrm{CH}_{2} \mathrm{CH}_{3}\right) ;{ }^{13} \mathrm{C}-\mathrm{NMR} \delta 164(\mathrm{C}=0), 152$ ( $\left.\delta \mathrm{C}=\right), 163,156$, 132, $113(\mathrm{Ph}), 115(\mathrm{CN}), 99(\mathrm{C}=), 67\left(\mathrm{OCH}_{2}\right), 21\left(\mathrm{OCH}_{2} \mathrm{CH}_{2}\right), 10$ $\left(\mathrm{OCH}_{2} \mathrm{CH}_{2} \mathrm{CH}_{3}\right)$; Anal. Calcd. for $\mathrm{C}_{13} \mathrm{H}_{11} \mathrm{ClFNO}_{2}: \mathrm{C}, 58.33 ; \mathrm{H}, 4.14 ; \mathrm{N}$, 5.23; Found: C, 59.46; H, 4.35; N, 5.58.

\section{Propyl 3-(2-chloro-6-fluorophenyl)-2-cyanoacrylate}

Yield 68\%; mp $50{ }^{\circ} \mathrm{C},{ }^{1} \mathrm{H}-\mathrm{NMR} \delta 8.1(\mathrm{~s}, 1 \mathrm{H}, \mathrm{CH}=)$ ) 7.4-6.7 $(\mathrm{m}, 3 \mathrm{H}, \mathrm{Ph}), 4.3\left(\mathrm{t}, 2 \mathrm{H}, \mathrm{OCH}_{2}\right), 1.7\left(\mathrm{~m}, 2 \mathrm{H}, \mathrm{OCH}_{2} \mathrm{CH}_{2}\right), 1.0(\mathrm{t}, 3 \mathrm{H}$, $\left.\mathrm{OCH}_{2} \mathrm{CH}_{2} \mathrm{CH}_{3}\right) ;{ }^{13} \mathrm{C}$-NMR $\delta 164(\mathrm{C}=0), 152(\mathrm{HC}=), 163,153,130$, 126, 119, $116(\mathrm{Ph}), 115(\mathrm{CN}), 92(\mathrm{C}=), 67\left(\mathrm{OCH}_{2}\right), 21\left(\mathrm{OCH}_{2} \mathrm{CH}_{2}\right)$, $10\left(\mathrm{OCH}_{2} \mathrm{CH}_{2} \mathrm{CH}_{3}\right)$; Anal. Calcd. for $\mathrm{C}_{13} \mathrm{H}_{11} \mathrm{ClFNO}_{2}$ : C, 58.33; $\mathrm{H}$, 4.14; N, 5.23; Found: C, 54.76; H, 4.65; N, 5.63.

\section{Propyl 3-(3-chloro-2-fluorophenyl)-2-cyanoacrylate}

Yield 73\%; mp $53{ }^{\circ} \mathrm{C},{ }^{1} \mathrm{H}-\mathrm{NMR} \delta 8.5$ (s, $\left.1 \mathrm{H}, \mathrm{CH}=\right)$ ) 8.3-7.2 $(\mathrm{m}, 3 \mathrm{H}, \mathrm{Ph}), 4.3\left(\mathrm{t}, 2 \mathrm{H}, \mathrm{OCH}_{2}\right), 1.7\left(\mathrm{~m}, 2 \mathrm{H}, \mathrm{OCH}_{2} \mathrm{CH}_{2}\right), 1.0(\mathrm{t}, 3 \mathrm{H}$, $\left.\mathrm{OCH}_{2} \mathrm{CH}_{2} \mathrm{CH}_{3}\right) ;{ }^{13} \mathrm{C}-\mathrm{NMR} \delta 163(\mathrm{C}=0), 152(\mathrm{HC}=), 162,153,130$, 126, 119, $116(\mathrm{Ph}), 115(\mathrm{CN}), 92(\mathrm{C}=), 67\left(\mathrm{OCH}_{2}\right), 21\left(\mathrm{OCH}_{2} \mathrm{CH}_{2}\right)$, $10\left(\mathrm{OCH}_{2} \mathrm{CH}_{2} \mathrm{CH}_{3}\right)$; Anal. Calcd. for $\mathrm{C}_{13} \mathrm{H}_{11} \mathrm{ClFNO}_{2}$ : C, 58.33; $\mathrm{H}$, 4.14; N, 5.23; Found: C, 58.17; H, 4.21; N, 5.25.

\section{Propyl 3-(3-chloro-4-fluorophenyl)-2-cyanoacrylate}

Yield 84\%; mp $118^{\circ} \mathrm{C},{ }^{1} \mathrm{H}-\mathrm{NMR} \delta 8.2(\mathrm{~s}, 1 \mathrm{H}, \mathrm{CH}=)$, 8.1-7.2 $(\mathrm{m}, 3 \mathrm{H}, \mathrm{Ph}), 4.3\left(\mathrm{t}, 2 \mathrm{H}, \mathrm{OCH}_{2}\right), 1.7\left(\mathrm{~m}, 2 \mathrm{H}, \mathrm{OCH}_{2} \mathrm{CH}_{2}\right), 1.0(\mathrm{t}, 3 \mathrm{H}$, $\left.\mathrm{OCH}_{2} \mathrm{CH}_{2} \mathrm{CH}_{3}\right) ;{ }^{13} \mathrm{C}-\mathrm{NMR} \delta 163(\mathrm{C}=0), 154(\mathrm{HC}=), 153,130,128$, 120, $117(\mathrm{Ph}), 115(\mathrm{CN}), 92(\mathrm{C}=), 67\left(\mathrm{OCH}_{2}\right), 21\left(\mathrm{OCH}_{2} \mathrm{CH}_{2}\right), 10$ $\left(\mathrm{OCH}_{2} \mathrm{CH}_{2} \mathrm{CH}_{3}\right)$; Anal. Calcd. for $\mathrm{C}_{13} \mathrm{H}_{11} \mathrm{ClFNO}_{2}: \mathrm{C}, 58.33 ; \mathrm{H}, 4.14 ; \mathrm{N}$, 5.23; Found: C, 58.18; H, 4.12; N, 5.10.

\section{Copolymerization}

The PPCA monomers when mixed with ethynylbenzene, $\mathrm{EB}$ at $\mathrm{EB} / \mathrm{PPCA}=3$ (mol) formed copolymers as indicated by white flaky precipitates in high excess of methyl alcohol. The conversion of the EB-PPCA copolymers was maintained 10 - 20\% to decrease compositional drift (Table 1). The PPCA content 8.4$25.8 \mathrm{~mol} \%$ in the copolymers indicated relatively high reactivity of the PPCA monomers towards EB radical Figure 1. 


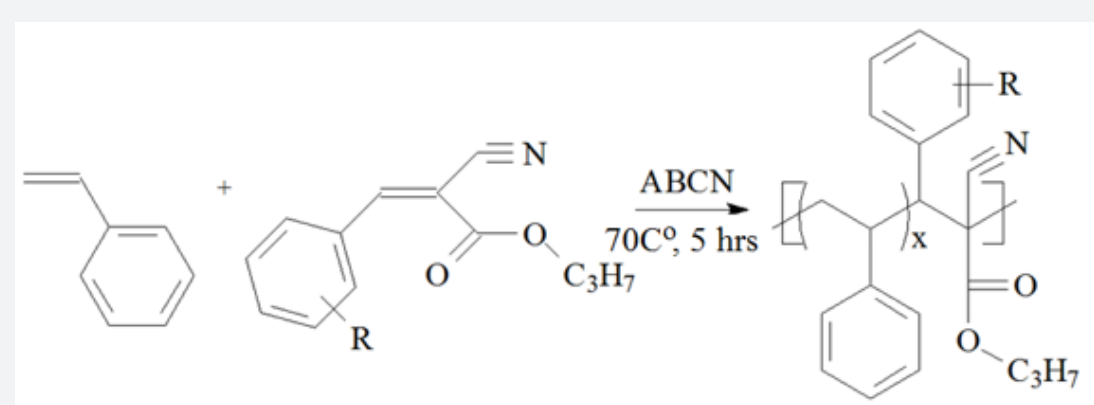

Figure 1: Copolymerization of ethynylbenzene and propyl 3-(R-phenyl)-2-cyanoacrylates, where $\mathrm{R}$ is 2-fluoro-5-methyl, 3-iodo-4-methoxy, 5-iodo-2-methoxy, 3,5-dichloro, 3,4-difluoro, 3,5-difluoro, 2-chloro-4-fluoro, 2-chloro-6-fluoro, 3-chloro-2-fluoro, and 3-chloro-4-fluoro.

Table 1: P(EB-co-PPCA) copolymers formationa.

\begin{tabular}{|c|c|c|c|c|c|c|c|c|}
\hline $\mathbf{R}$ & $\begin{array}{c}\text { Conversion } \\
\text { wt } \%\end{array}$ & $\begin{array}{c}\text { Nitrogen } \\
\text { wt } \%\end{array}$ & $\begin{array}{c}\text { \% Mole } \\
\text { PPCA }\end{array}$ & MW (kD) & $\operatorname{Tg}\left({ }^{\circ} \mathrm{C}\right)$ & $\begin{array}{c}\text { Onset of } \\
\text { Decomp. }\left({ }^{\circ} \mathrm{C}\right)\end{array}$ & $\begin{array}{c}50 \mathrm{wt} \% \text { loss } \\
\left({ }^{\circ} \mathrm{C}\right)\end{array}$ & Residue wt $\%$ \\
\hline 2-F-5- $\mathrm{CH}_{3}$ & 18.3 & 2.78 & 28.8 & 26.3 & 124 & 255 & 336 & 2 \\
\hline 3-I-4- $\mathrm{OCH}_{3}$ & 12.8 & 1.85 & 21.2 & 23.9 & 103 & 135 & 338 & 2 \\
\hline $5-\mathrm{I}-2-\mathrm{OCH}_{3}$ & 15.8 & 2.17 & 27.5 & 11 & 111 & 207 & 340 & 3 \\
\hline $3,5-\mathrm{Cl}_{2}$ & 17.6 & 2.69 & 30.5 & 48.1 & 151 & 252 & 327 & 2 \\
\hline $3,4-\mathrm{F}_{2}$ & 14.7 & 2.84 & 30.1 & 36.2 & 138 & 171 & 331 & 1 \\
\hline $3,5-\mathrm{F}_{2}$ & 17.6 & 2.67 & 27.6 & 32.1 & 112 & 205 & 345 & 2 \\
\hline 2-Cl-4-F & 13.8 & 2.53 & 27.9 & 29.3 & 121 & 207 & 349 & 3 \\
\hline 2-Cl-6-F & 12.2 & 1.71 & 15.9 & 27.9 & 112 & 203 & 361 & 2 \\
\hline $3-\mathrm{Cl}-2-\mathrm{F}$ & 17.8 & 2.67 & 28.9 & 30.2 & 112 & 210 & 369 & 1 \\
\hline 3-Cl-4-F & 12.2 & 2.65 & 28.8 & 28.1 & 110 & 190 & 362 & 1 \\
\hline
\end{tabular}

Note: ${ }^{a}$ EB/PPCA: 3 (mol) / methyl benzene $/ 70^{\circ} \mathrm{C} / 5 \mathrm{hrs}$. bby GPC.

The EB-PPCA copolymers were soluble in tetrahydrofuran, ethyl acetate, chloroform and dimethylformamide, and insoluble in methyl alcohol, ethyl and petroleum ethers. GPC analysis the copolymers indicated weight-average molecular masses 10.727.8kD (Table 1). According to elemental analysis, between 18.3 \& $42.6 \mathrm{~mol} \%$ of PPCA monomer is present in the copolymers prepared at EB/PPCA $=3(\mathrm{~mol})$, which is indicative of relatively high reactivity of the monomers towards EB. Results of DSC and TGA analyses are presented in Table 1.

The FTIR spectra of the monomers were compared with those of copolymers and poly(ethynylbenzene) thus providing evidence that the reaction between the PPCA monomers and EB is a copolymerization. The spectra of the copolymers show overlapping bands in $3200-2820 \mathrm{~cm}^{-1}$ region corresponding to $\mathrm{C}-\mathrm{H}$ stretch vibrations. The bands for the PPCA monomer unit are 2246-2238 (w, CN), 1752-1733 (s, C=0), and 1252-1226 $\mathrm{cm}^{-1}$ (m, C-0). Benzene rings of both monomers show ring stretching bands at $1500-1400 \mathrm{~cm}^{-1}$ as well as a doublet $824-715 \mathrm{~cm}^{-1}$, associated with $\mathrm{C}-\mathrm{H}$ out of plane deformations. These bands can be readily identified in ethynylbenzene copolymers with TSE monomers containing cyano and carbonyl electron withdrawing groups.
EB-PPCA copolymers microstructure analysis is based on 1H and 13C NMR spectroscopy (DEPT, HETCOR, NOESY and JMODXH) of EB copolymers with 2-phenyl-1,1-dicyanoethene [9] which showed the formation of both head-to-tail and headto-head alternating monomer structures, as well as short EB sequences.

\section{Conclusion}

Novel phenyl-disubstituted propyl 2-cyano-3-phenyl-2-propenoates were prepared and copolymerized with ethynylbenzene. The compositions of the copolymers were calculated from nitrogen analysis and the structures were analyzed by IR, H1 \& 13C-NMR. The thermal gravimetric analysis indicated that the copolymers decompose in in two steps, first in the $200-500^{\circ} \mathrm{C}$ range with residue (1-3\%wt), which then decomposed in the 500-800 ำ range.

\section{References}

1. Buschauer, Friese Kimmel A, Baumann G, Schunack W (1992) Synthesis and histamine H2 agonistic activity of arpromidine analogues: replacement of the pheniramine-like moiety by non-heterocyclic groups. European Journal of Medicinal Chemistry 27(4): 321-330.

2. Elinson MN, Vereshchagin AN, Korshunov AD, Ryzhkov FV, Egorov MP (2017) Heterocyclic Communications 23: 85. 
3. Das S, Yasmin H, Mehedi MM, Roy SC, Nahar L, et al. (2008) Total synthesis and analgesic activity of 6-fluoroindan-1-carboxylic acid. Tetrahedron 64(37): 8642-8645

4. Duffy C, Zetterlund PB, Aldabbagh F (2018) Radical Polymerization of Alkyl 2-Cyanoacrylates. Molecules 23(2): 465

5. Kharas GB, Chavez SE, Luna AN, Lusk EE, Mendez DP, et al. (2014) Macromol Sci Part A: Pure and Applied Chemistry 51: 1.

6. Kharas GB, Molina ES, Fitzpatrick BM, Francis MC, Gallardo C, et al. (2013) J Macromol Sci Part A: Pure and Applied Chemistry, 50: 370.
7. Kharas GB, Schjerven WS, Maurer LM, McGee GC, McGovern ME, et al. (2016) J Macromol Sci Part A: Pure and Applied Chemistry 53: 258.

8. Smith MB, March J (2001) Addition to Carbon-Hetero Multiple Bonds, In March's Advanced Organic Chemistry J Wiley \& Sons: New York, Ch16, p. 1225.

9. Kharas GB, Murau PA, Watson K, Harwood HJ (1992) Microstructure of styrene copolymers with 2-phenyl-1,1-dicyanoethene. Polym Int 28(1): 67-74

\section{Your next submission with Juniper Publishers will reach you the below assets}

- Quality Editorial service

- Swift Peer Review

- Reprints availability

- E-prints Service

- Manuscript Podcast for convenient understanding

- Global attainment for your research

- Manuscript accessibility in different formats

( Pdf, E-pub, Full Text, Audio)

- Unceasing customer service

Track the below URL for one-step submission https://juniperpublishers.com/online-submission.php 